\title{
HOMO IMITANS IS HOMO LOQUENS. ON VOCAL IMITATION
}

\author{
JOANA ROSSELLÓ \\ joana.rossello@ub.edu \\ Department of Catalan Philology and General Linguistics, Barcelona, Spain
}

Words are likely the most specifically linguistic ingredient of human language. Although conventional, rather than natural, they are, however, nature-culture hybrids since their acquisition depends on a biologically shared capacity for vocal imitation (VI). VI is present in vocal production learning, a scarce trait among animals that in primates is only shown in humans, the single homo loquens. By relating VI abilities, which are essential to build our huge vocabularies, to a biologically grounded vocal learning capacity in humans, the present proposal opposes to the view that VI is a developmental instance of a previous multimodal general imitative capacity (Donald, 1993) which would be central, innately given and unique to our species, thus seen as homo imitans (Meltzoff \& Moore, 1997). Albeit recognizing the centrality of imitation in cooperative social interaction and cultural learning, I will argue to the contrary that VI has primacy and is co-opted to support general imitation. This view, besides solving the general neglect of VI in the literature on human imitation, may mediate between the two dominant psychological models of imitation, is parsimoniously explanatory, makes right empirical predictions regarding the signed modality and conditions such as autism and, finally, puts an inherently ostensive communication rather than action at the basis of human cognition. The Basic Vocal Imitation (BVI) approach might enlighten the debate between the Associative Sequence Learning (ASL) model (Catmur et al. 2009, Heyes 2016) and the Active Intermodal Matching (AIM) model (Meltzoff \& Moore, 1997) underlying the homo imitans view. As a complement to ASL, BVI might provide a well-founded empirical basis for the ASL position that imitation mechanisms in humans are shared with animals. Besides, BVI circumvents the "correspondence problem" which goes with the mapping between "felt but unseen movements of the self with the seen but unfelt movements of the other" 
(Heyes, 2016). Indeed, VI avoids the correspondence problem associated with the imitation of visual displays since one hears their own vocalizations as a result of the total feedback which is intrinsic and unique to vocal-auditory communication (Fitch, 2005). VI, on the other hand, is overtly developing by 8 months, as babbling unequivocally shows. In contrast, evidence for neo-natal non-vocal imitation is becoming increasingly weaker (Oostenbroek et al. 2016), which adds to the argument that the highly skilled imitation human abilities may be built on top of VI. Also overimitation, i.e., the imitation of actions that are causally irrelevant, is enlightened: it would be a uniquely human phenomenon among primates (Clay \& Tennie, 2017) because humans are the only primates with vocal learning abilities and all vocal learners imitate arbitrary sequences of sounds, which in humans are in turn linked to arbitrary meanings in words.

If VI is basic, speech must be default. Sign is not default, it goes with deafness; and overlapping neural correlates for both speech and sign seen in native deaf signers arise from brain plasticity operating in sensory deprivation rather than being evidence for an alleged modality-independence of language. That the overlap lies in auditory areas in the brain is expected from this perspective. Regarding imitation in autism (ASD), Vivanti \& Hamilton's (2014) review has shown that autistic individuals imitate less and worse than neurotypicals, mainly if the actions are sequential and not goal-directed (i.e. overimitation). Among explanatory proposals for this autistic deficit, Vivanti \& Hamilton (2014) conclude that the sensorimotor deficit theory seems to be superior to the rest (e.g. 'failed direct self-other mapping' and other top-down proposals). Crucially, these authors do not mention either non-verbal autism ( $25 \%$ of ASD) or any deficit in VI, with the latter being inherent to the former. Yet, by putting an abnormal vocal learning capacity, with a consequent VI deficit, at the basis of ASD, the general imitative impairment as observed in the condition falls into place. The superiority of the sensorimotor deficit account also follows since sensorimotor integration is essential to VI. Finally, that nonverbal autistics can understand intentional actions and learn to communicate imperatively through images (PECS) rather than gestures, in which they are also impaired, strongly suggests that, contrary to the position in Scott-Phillips (2015), ostensive and declarative communication is primarily auditory and exapted to gestures.

\section{References}

Heyes, C. (2016). Homo imitans? Seven reasons why imitation couldn't possibly be associative. Philosophical Transactions of the Royal Society B: Biological Sciences, 371(1686) 
Meltzoff AN. (1988) The human infant as Homo imitans. In Social learning: psychological and biological perspectives (eds TR Zentall, BG Galef ), 319-341. NJ: Erlbaum.

Vivanti, G., \& Hamilton, A. (2014). Imitation in ASD. Handbook of Autism and Pervasive Developmental Disorders, Vol. 1. Diagnosis, Development, and Brain Mechanisms, ed. By Volkmar et al., 278-302. 